\title{
In Situ Lorentz Electron Microscopy Imaging of Skyrmions in Geometric Confined Structures
}

\author{
N. Bagués ${ }^{1 *}$, B. D. Esser ${ }^{1}$, A. S. Ahmed ${ }^{2}$, J. Rowland ${ }^{2}$, J.-Q. Yan $^{3}$, D. E. Huber ${ }^{1}$, M. Randeria ${ }^{2}$, D. W. \\ $\mathrm{McComb}^{1,4}$ \\ 1. Center for Electron Microscopy and Analysis, The Ohio State University, Columbus, OH, USA \\ 2. Department of Physics, The Ohio State University, Columbus, OH, USA \\ 3. Materials Science and Technology Division, Oak Ridge National Laboratory, Oak Ridge, TN, USA. \\ 4. Department of Materials Science and Engineering, The Ohio State University, Columbus, OH, USA \\ * Corresponding author: baguessalguero.2@osu.edu
}

Magnetic skyrmions, which are topologically stable spin textures, have attracted special attention in recent years due to their potential for use in the next generation of data storage devices [1]. However, in order to design and create future memory devices, it is essential to understand skyrmion stability in confined nanostructures. Recent studies have reported the observation of skyrmion chains in nanostripes [2], deformation of skyrmions in wedge-shaped nanostripes [3], and the stabilization of single skyrmions in a corral of artificial surface pits [4]. In these studies, magnetic contrast imaging using electron microscopy (Lorentz transmission electron microscopy (LTEM) [2], electron holography, or [3] differential phase contrast (DPC) scanning (S)TEM [4]) was essential to study the effects of geometrical confinement on the skyrmion phase with high real space resolution.

In this work, we study the stabilization of skyrmions in geometrically confined FeGe specimens by in-situ Lorentz electron microscopy imaging. FeGe bulk is a chiral magnet with a helical transition at a Curie temperature of $T_{\mathrm{c}} \sim 280 \mathrm{~K}$ [5]. A focused ion beam (FIB) instrument was used to create confined regions in bulk FeGe by milling channels with the ion beam to leave nano-strips of material of various widths and thicknesses. High angle annular dark field (HAADF) STEM and electron energy loss spectroscopy (EELS) was used to measure width and thickness variations of the nano-strips, respectively. Variable magnetic field (from 0 to $950 \mathrm{Oe}$ ) Lorentz TEM was used to visualize and study the magnetic phases in the confined regions (nano-strips) and non-confined regions. Lorentz TEM uses Fresnel contrast in through-focal image series to observe the magnetic contrast. The sharp interface between nano-strips and the vacuum may lead to strong fringe contrast around the edges, making quantification a challenge. To avoid such artifacts, Lorentz STEM imaging using a segmented annular detector (DPC STEM) and EMPAD pixelated detector (4D STEM) is performed. Lorentz STEM techniques have the advantage over conventional Lorentz TEM of being performed in-focus, thus reducing resolution-limiting artifacts.

Figure 1 shows a STEM image of a geometrically confined FeGe specimen with the channels (black) and the nano-strips ranging in width from 50-500 nm (gray), together with an EELS thickness map showing the variation in thickness from $80-130 \mathrm{~nm}$ across the same area of the sample. We observe the formation of the helical phase across the entire sample at zero magnetic field and $265 \mathrm{~K}$. As the applied perpendicular magnetic field is increased, we observe the formation of skyrmions in the non-confined region and in some regions of the nano-strips. Figure 2 shows a Lorentz TEM image reconstruction after processing via the transport of intensity equation (TIE) showing the hexagonal lattice of skyrmions. The regions of material that have been purposefully shaped into nano-strips (lower left, Figure 2), strongly affects the orientation of the skyrmion lattice in a way that most efficiently packs skyrmions. This is in contrast to the non-confined regions (upper right, Figure 2) where the skyrmion lattice is rotated with respect to the 
nano-strips. At the interface between these two orientations, the hexagonal skyrmion lattice is distorted by dislocations, resulting in skyrmions with 5 and 7 nearest neighbors (inset Figure 2). As more field is applied, the effect of geometric confinement is strong enough to rotate the non-confined skyrmion lattice to match the orientation of the lattice within the nano-strips. These results point the ability to control the orientation of the skyrmion lattice via geometrical confinement, which may influence the future design of devices. [6]

\section{References:}

[1] N. Nagaosa and Y. Tokura, Nature Nanotechnology 8 (2013), p. 899-911.

[2] H. F. Du et al., Nature Communications 6, (2015), 8504.

[3] C. Jin et al., Nature Communications 8, (2017), 15569.

[4] T. Matsumoto et al., Nano Letters 18(2), (2018), p. 754-762.

[5] X. Z. Yu et al., Nature Materials 10, (2011), p 106-109.

[6] The authors acknowledge funding from Defense Advanced Research Projects Agency (DARPA) under Grant No. D18AP00008 . Crystal growth and characterization at ORNL was supported by the U.S. Department of Energy (DOE), Office of Science, Basic Energy Sciences (BES), Materials Sciences and Engineering Division.
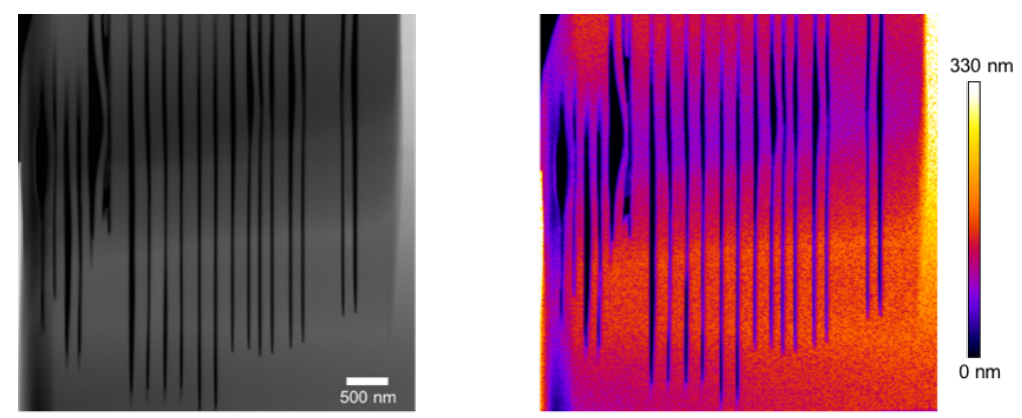

Figure 1. Left, HAADF-STEM image of a bulk FeGe specimen with nano-strips (gray) and the channels (black). Right, thickness map obtained using EELS from the same area showing variations in thickness across the nano-strips from $\sim 80 \mathrm{~nm}$ (purple) to $\sim 130 \mathrm{~nm}$ (orange-red).
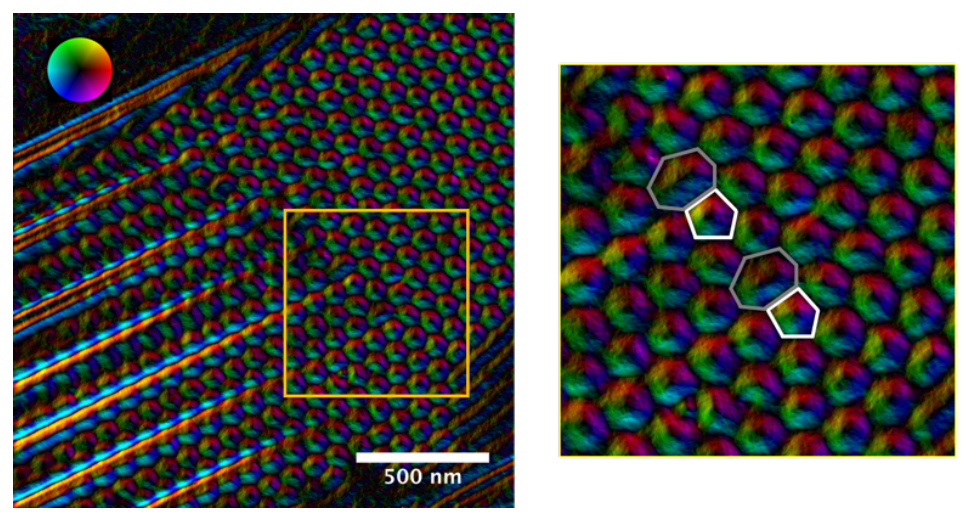

Figure 2. Left, Lorentz TEM image reconstruction after processing via TIE of a geometrically confined FeGe specimen $(450 \mathrm{Oe}, 265 \mathrm{~K})$. Color in the image corresponds to the direction of the in-plane magnetic induction, as indicated by the wheel at the top left. The orange square marks the magnified area presented on the right, showing defects in the hexagonal lattice, resulting in skyrmions with 7 and 5 nearest neighbors (grey and white, respectively). 